\title{
Determination of Ion Mobility Collision Cross Sections for Unresolved Isomeric Mixtures Using Tandem Mass Spectrometry and Chemometric Deconvolution
}

\author{
Brett Harper ${ }^{1}$, Elizabeth K. Neumann ${ }^{2, \phi}$, Sarah M. Stow ${ }^{3,4,5,6}$, Jody C. May ${ }^{3,4,5,6}$, John A. \\ McLean $^{3,4,5,6}$, and Touradj Solouki ${ }^{2 *}$ \\ ${ }^{1}$ Institute of Biomedical Studies, Baylor University, Waco, TX 76798, USA \\ ${ }^{2}$ Department of Chemistry and Biochemistry, Baylor University, Waco, TX 76798, USA \\ ${ }^{3}$ Department of Chemistry, Vanderbilt University, Nashville, TN 37235, USA \\ ${ }^{4}$ Vanderbilt Institute of Chemical Biology, Nashville, TN 37235, USA \\ ${ }^{5}$ Vanderbilt Institute for Integrative Biosystems Research and Education, Nashville, TN 37235, \\ USA \\ ${ }^{6}$ Center for Innovative Technology, Nashville, TN, 37235, USA
}

\begin{abstract}
Ion mobility (IM) is an important analytical technique for determining ion collision cross section (CCS) values in the gas-phase and gaining insight into molecular structures and conformations. However, limited instrument resolving powers for IM may restrict adequate characterization of conformationally similar ions, such as structural isomers, and reduce the accuracy of IM-based CCS calculations. Recently, we introduced an automated technique for extracting "pure" IM and collision-induced dissociation (CID) mass spectra of IM overlapping species using chemometric deconvolution of post-IM/CID mass spectrometry (MS) data [J. Am. Soc. Mass Spectrom., 2014, 25, 1810-1819]. Here we extend those capabilities to demonstrate how extracted IM profiles can be used to calculate accurate CCS values of peptide isomer ions which are not fully resolved by IM. We show that CCS values obtained from deconvoluted IM spectra match with CCS values measured from the individually analyzed corresponding peptides on uniform field IM instrumentation. We introduce an approach that utilizes experimentally determined IM arrival time (AT) "shift factors" to compensate for ion acceleration variations during post-IM/CID and significantly improve the accuracy of the calculated CCS values. Also, we discuss details of this IM deconvolution approach and compare empirical CCS values from traveling wave (TW)IMMS and drift tube (DT)IM-MS with theoretically calculated CCS values using the projected superposition approximation (PSA). For example, experimentally measured deconvoluted TWIM-MS mean CCS values for doubly-protonated RYGGFM, RMFGYG, MFRYGG, and FRMYGG were $288.8 \AA^{2}, 295.1 \AA^{2}, 296.8 \AA^{2}$, and $300.1 \AA^{2}$; all four of these CCS values were within $1.5 \%$ of independently measured DTIM-MS values.
\end{abstract}

\author{
AUTHOR INFORMATION \\ ${ }^{中}$ Current Address: Department of Chemistry, University of Illinois at Urbana-Champaign, \\ Urbana, IL 61801, USA \\ Corresponding Author \\ *E-mail: Touradj_Solouki@baylor.edu. Telephone: 254-710-2678
}


Keywords: peak deconvolution, chemometric analysis, resolving power, structural isomers, collision-induced dissociation (CID), ion mobility-mass spectrometry

Abbreviations: arrival time (AT), automated ion mobility deconvolution (AIMD), collision cross section (CCS), confidence level (C.L.), drift tube ion mobility-mass spectrometry (DTIMMS), extracted ion drift time distribution (EIDTD), ion mobility (IM), ion mobility-mass spectrometry (IM-MS), projected superposition approximation (PSA), root-mean-squared deviation (RMSD), SIMPLe-to-use Interactive Self-modeling Mixture Analysis (SIMPLISMA), traveling wave ion mobility-mass spectrometry (TWIM-MS) 


\section{Introduction}

Ion mobility-mass spectrometry (IM-MS) is an important analytical technique for differentiating the structure(s) and conformation(s) of gas-phase ions [1, 2]. IM-MS can be used to rapidly separate ions based on their gas-phase sizes and shapes, while collision cross-section (CCS) values (determined from the IM drift times) can aid molecular identification and be used to gain insight into the size and folding of molecules. There is compelling evidence that structural insights obtained from IM-MS studies may be relevant to solution-phase structures and chemistry [3-9]. Several commercial IMS and IM-MS systems are available which are capable of determining CCS values; the most prevalent of these measurements are based on drift tube (DT)IM-MS and traveling wave (TW)IM-MS technologies [1, 2]. CCS measurements in DTIMMS are relatively straightforward because ion drift times $\left(t_{D}\right)$ are directly proportional to their CCS $[2,10]$. However, in TWIM-MS (where a non-uniform electric field is used to move ions) ion CCS values are proportional to ${t_{D}}^{x}$, where " $x$ " is an empirically derived parameter that must be calculated from analysis of calibrants with known CCS values [11].

Under a given set of experimental conditions, the ability to resolve isomeric species is principally governed by two fundamental properties, namely: IM resolving power and IM resolution. The IM resolving power (i.e., arrival time (AT) $/ \Delta \mathrm{AT}_{50 \%}$ or $\mathrm{CCS} / \Delta \mathrm{CCS}_{50 \%}$; where $\triangle \mathrm{AT}_{50 \%}$ and $\triangle \mathrm{CCS}_{50 \%}$ are the IM peak widths (measured in seconds and CCS, respectively at half-height) defines the peak width of a single-component [10, 12-15]. Relatedly, the IM resolution (i.e., $\left[\mathrm{AT}_{1}-\mathrm{AT}_{2}\right] /\left[\Delta \mathrm{AT}_{1}+\Delta \mathrm{AT}_{2}\right]$; where $\mathrm{AT}_{1}$ and $\mathrm{AT}_{2}$ are the centroid arrival times of two ion populations and $\Delta \mathrm{AT}_{1}$ and $\Delta \mathrm{AT}_{2}$ are the base widths of two ion populations) defines the ability to distinguish two closely eluding ion populations [10, 12]. Continuous improvements in achievable resolving power and resolution of contemporary instrumentation have extended the 
ability to differentiate isomeric systems. To improve IM peak separation, several groups have focused on different strategies including using alternative drift gasses or gas modifiers [16-21], developing new instruments [22-27], or customizing existing systems [28]. Additional improvements in software and post-data acquisition approaches can also enhance data interpretation for convoluted IM peaks. For instance, to circumvent instrumental limitations, some groups have used post-IM fragmentation and assigned IM profiles of diagnostic fragment ions to IM-unresolved precursor ions [29-35]. For example, similar to spectral deconvolution of overlapping gas chromatography (GC)/MS peaks [36], Clemmer's group demonstrated that isomers with unresolved drift time distributions could be differentiated by analyzing extracted ion drift time distributions (EIDTDs) of unique fragment ions generated from post-DTIM collision-induced dissociation (CID) [31, 32]. Recently, Khakinejad et al. demonstrated that EIDTDs from post-DTIM/electron transfer dissociation data could be used to calculate CCS values of IM-unresolved peptide conformers [34]. These post-IM/CID approaches demonstrated that fragment ions could be used to differentiate unresolved isomers present in a mixed AT; however, to date, there have not been any attempts at reconstructing pure AT profiles from convoluted IM spectra for determination of CCS values for IM-overlapping ions.

Previously, we introduced a chemometric data deconvolution technique [37] based on SIMPLe-to-use Interactive Self-modeling Mixture Analysis (SIMPLISMA) [38] which allowed the extraction of "pure" IM and post-IM/CID mass spectra for IM-unresolved species. Subsequently, we integrated SIMPLISMA into an automated IM deconvolution (AIMD) software, which improved the speed and accuracy of IM-MS analyses [39]. Moreover, we showed that chemical rank determination techniques [40,41] could be used to identify the 
number of IM-unresolved species without prior knowledge of the sample composition [37, 39, 42-44].

In this manuscript, we demonstrate another unique utility of chemometric deconvolution of post-IM/CID MS data; specifically, we show that CCS values of IM-unresolved species, such as reverse sequence pentapeptide isomers (i.e., SDGRG and GRGDS) and scrambled sequence enkephalin hexapeptide isomers (i.e., RYGGFM, RMFGYG, MFRYGG, and FRMYGG), can be accurately calculated. In this method, we utilize AT "shift factors" to compensate for effects of electric field variations during post-IM/CID and reduce differences between mean calculated CCS values for IM deconvoluted profiles and CCS values obtained from individually measured analytes. Finally, we compare our deconvoluted TWIM-MS CCS values for enkephalin hexapeptide isomers to (a) experimental values obtained by using DTIM-MS and (b) theoretical values calculated by using the projected superposition approximation (PSA).

\section{Materials and methods}

\subsection{Sample preparation}

Two reverse pentapeptide isomers (amino acid sequences: SDGRG and GRGDS) and poly-alanine (used as the CCS calibrant for TWIM-MS analysis) [11, 45] were purchased from Sigma-Aldrich (St. Louis, MO, USA) and [Arg0] Met-enkephalin (amino acid sequence: RYGGFM) was purchased from American Peptide (Sunnyvale, CA, USA). Three sequencescrambled enkephalin hexapeptides (amino acid sequences: RMFGYG, MFRYGG, and FRMYGG) were custom synthesized by Peptide 2.0, Inc. (Chantilly, VA, USA) and their identities were confirmed by CID MS analyses (supporting information, Figure S1). The pentapeptide mixture was chosen because of its previous use in characterizing the IM resolving

powers of the Synapt HDMS TWIM-MS instruments [28]. The four hexapeptide isomers were 
selected as model systems because of their overlapping IM profiles; moreover, to the best of our knowledge, CCS values of these encephalin hexapeptides have not been previously characterized. Optima grade acetic acid and methanol were purchased from Fisher-Scientific (Waltham, MA, USA). Water was purified using a Direct-Q 3 UV water purification system (EMD Millipore Corporation, Billerica, MA, USA). Peptides were prepared by reconstitution in a water: methanol: acetic acid (49.95: 49.95: 0.1) solvent composition at final analyte concentrations of $\sim 1 \mu \mathrm{M}$. Peptide mixtures contained approximately equimolar concentrations of each isomer.

\subsection{Ion mobility-mass spectrometry}

TWIM-MS experiments were performed as previously described [37, 39, 42-44, 46] using Synapt G1 (for the pentapeptide binary mixture) and G2-S (for the hexapeptide quaternary mixture) systems (Waters Corporation, Milford, MA, USA). Experimental details regarding the collection of TWIM-MS data reported in this manuscript are included in the supporting information (Table S1). Drift tube CCS measurements were obtained on a commercial ESI-IMQTOF Agilent 6560 (Agilent Technologies, Santa Clara, CA, USA) as previously described [47, 48]; briefly, buffer gas (i.e., nitrogen $\left(\mathrm{N}_{2}\right)$ ) was maintained at a pressure of $\sim 4$ Torr and drift voltages were varied to correct for the non-IM flight time of the ions through interfacing ion optics. Agilent tune mix was run with each hexapeptide sample as an internal CCS standard.

\subsection{Data analysis}

Post-IM/CID MS data were deconvoluted using automated IM deconvolution (AIMD) software developed in-house [39], which utilizes the SIMPLISMA algorithm [38] to extract "pure" IM and mass spectra. The nominal $\mathrm{m} / \mathrm{z}$ values with the highest corresponding "purity values" (used to guide deconvolution) [37] for deconvoluted singly-protonated SDGRG and 
GRGDS (i.e., $[\mathrm{SDGRG}+\mathrm{H}]^{+}$and $[\mathrm{GRGDS}+\mathrm{H}]^{+}$, respectively) were $m / z 289\left(\right.$ i.e., $\mathrm{y}_{3}{ }^{+}$) and $\mathrm{m} / \mathrm{z}$ 386 (i.e., $\mathrm{b}_{4}^{+}$), respectively. Likewise, the nominal $\mathrm{m} / \mathrm{z}$ values with the highest corresponding "purity values" for deconvoluted doubly-protonated RYGGFM, RMFGYG, MFRYGG, and FRMYGG (i.e., $[\mathrm{RYGGFM}+2 \mathrm{H}]^{2+}$, $[\mathrm{RMFGYG}+2 \mathrm{H}]^{2+},[\mathrm{MFRYGG}+2 \mathrm{H}]^{2+}$, and $[\mathrm{FRMYGG}$ $+2 \mathrm{H}]^{2+}$, respectively) were $m / z 150$ (i.e., $\left.\mathrm{y}_{1}{ }^{+}\right), m / z 492\left(\right.$ i.e., $\left.\mathrm{b}_{4}{ }^{+}\right), m / z 599\left(\right.$ i.e., $\left.\mathrm{y}_{5}^{+}\right)$, and $m / z 133$ $\left(\right.$ i.e., $\left.\mathrm{y}_{2}^{+}\right)$, respectively. All IM arrival times are obtained from the raw data (i.e., poly-alanine, individually run peptides, and peptide mixtures) and correspond to the experimental centroid values of the AT extracted from the $\mathrm{m} / \mathrm{z}$ of the monoisotopic (viz., ${ }^{12} \mathrm{C}_{\text {all }}$ ) peak using a $\pm 0.03 \mathrm{~m} / \mathrm{z}$ mass range (e.g., $\mathrm{m} / \mathrm{z} 491.22 \pm 0.03$ for $\left.[\mathrm{SDGRG}+\mathrm{H}]^{+}\right)$. Note that the term "arrival time" (or "AT") is used throughout this manuscript (including figures and supporting information files) to denote "raw" times that have not been corrected for charge state, mass, or time spent outside of the IM cell (e.g., in the transfer cell or TOF). Post-IM/CID MS data were analyzed with AIMD software with averages of 10 minutes (588 scans; pentapeptides) or 1 minute acquisition times (58 scans; enkephalin hexapeptides). All other TWIM data were collected for 1 minute acquisition time (58 scans), unless otherwise noted. DTIM-MS experiments were collected for 2 minutes acquisition time (108 scans). All experiments were performed a minimum of three times; all reported errors are at the 95\% confidence level (C.L.). Experimental TWIM-MS CCS values were calculated using the method proposed by Ruotolo and coworkers.[11] The MasonSchamp equation [49] was used to determine experimental CCS values from ion drift times for all DTIM-MS experiments. Microsoft Excel (Office 2010, Microsoft Corp., Redmond, WA, USA) spreadsheets containing CCS calculations (including insignificant figures), AT corrections, and statistical tests can be found in the supporting information (viz., Spreadsheet S1 
corresponds to the pentapeptides, Spreadsheet S2 corresponds to the TWIM-MS hexapeptide measurements, Spreadsheet S3 corresponds to the DTIM-MS hexapeptide measurements).

\subsection{Theoretical CCS calculations}

Theoretical CCS values for the four enkephalin hexapeptide isomers (i.e., RYGGFM, RMFGYG, MFRYGG, and FRMYGG) were calculated using a simulated annealing protocol implemented with the AMBER software [50]. The doubly-protonated hexapeptide isomers were constructed using standard amino acid templates in the xLEaP module such that protonation sites were at the arginine and N-termini. Because xLEaP contains amino acid templates that do not incorporate hydrogens on C-terminal residues, these were constructed manually and subsequently geometry optimized at the Hartree-Fock level with a 6-31G* basis set using Gaussian 09 [51]. The hexapeptide isomers were then energy minimized with the sander module [50], and subsequently heated to $700-800 \mathrm{~K}$ over a 10 ps molecular dynamics simulation. An extended molecular dynamic simulation was run at 700-800 $\mathrm{K}$ for 9,000 ps and structural snapshots were saved every 3 ps throughout the simulation, resulting in 3,000 unique structures. These structures were cooled to $300 \mathrm{~K}$ during 15 ps molecular dynamics simulations resulting in low-energy conformations that were used for theoretical CCS determination [52, 53].

A combination of MOBCAL [54-57] and projected superposition approximation (PSA) [58-61] were used to calculate the theoretical CCS values. Helium (He) values were obtained in MOBCAL using the projection approximation. PSA was then used to calculate nitrogen $\left(\mathrm{N}_{2}\right)$ CCS values for a series of low-energy structures ( 20) spanning the entire CCS range for which the structures were obtained. A linear function was determined from these values to convert the remaining $\mathrm{He} C \mathrm{CS}$ values to $\mathrm{N}_{2} \mathrm{CCS}$ values as previously demonstrated [52]. PSA CCS values 
were calculated using the online service hosted by the University of California, Santa Barbara [62].

\section{Results and discussion}

Figure 1 (black) shows the IM profile of a mixture of two isomeric reverse pentapeptides (i.e., $[\mathrm{SDGRG}+\mathrm{H}]^{+}$and $[\mathrm{GRGDS}+\mathrm{H}]^{+}$at $m / z$ 491.22). As previously reported, the IM resolving power of the Synapt G1 is insufficient to separate these two peptides [28, 39]. Characterization of unknown samples with overlapping IM profiles (such as those shown in Figure 1, black IM profile) would be challenging, as IM-unresolved peaks could erroneously be assigned to a single analyte. Particularly in this example, the mixture exhibits a symmetric Gaussian-like IM profile indicative of what would be obtained from a single analyte. Presuming a single IM peak for this binary mixture would lead to measurement of a CCS corresponding to a mixture of two analytes. In the following sections, we show that chemometric deconvolution of post-IM/CID MS data can be used to extract ATs of IM-unresolved isomers that can be used to calculate accurate CCS values. Because of ion acceleration during post-IM/CID, ATs of deconvoluted IM profiles are shorter than ATs of individually analyzed species without postIM/CID (i.e., obtained under low-energy conditions). Here, we demonstrate that corrected ATs can be used for deconvoluted IM spectra to improve experimentally calculated CCS values.

\subsection{Chemometric deconvolution}

Using our previously reported AIMD software,[39] the unresolved IM profile in Figure 1 (black IM profile) was deconvoluted to yield extracted IM profiles for $[\text { SDGRG }+\mathrm{H}]^{+}$and $[\text { GRGDS }+\mathrm{H}]^{+}$(green and red IM spectra in Figure 1, respectively). To check the validity of the deconvolution, we compared constructed CID mass spectra for each peptide from deconvolution of the data in Figure 1 to CID mass spectra of individually analyzed peptides (supporting 
information, Figure S2). The degree of similarity between deconvoluted and pure CID mass spectra were assessed using a previously described matching score $(R)$ algorithm [46]:

$$
\begin{aligned}
& \text { Equation } 1 \\
& \qquad R=1-\frac{\sum_{m=1}^{n}\left|x_{m}-x_{m}^{\prime}\right|}{\sum_{m=1}^{n}\left(x_{m}-x_{m}^{\prime}\right)}
\end{aligned}
$$

where $n$ is the total number of fragment ion $m / z$ values, $m$, with $x_{m}$ and $x_{m}^{\prime}$ as fragment ion intensities (for a given value of $m$ ) for pure and deconvoluted spectra, respectively. $R$ values of 0 and 1 indicate no correlation and a perfect match, respectively [46]. An $R$ value of $\geq 0.75$ was considered a "successful" threshold for our deconvolution [46]. Calculated $R$ values for all deconvoluted reverse peptide isomer CID mass spectra were $>0.86$. Moreover, cross-validation matching scores $\left(R^{\prime}\right)$ were calculated by comparing the deconvoluted CID mass spectra for each peptide isomer with the reference spectrum for the non-matching isomer. The highest calculated $R$ ' value was $\sim 0.46$ (i.e., indicating "unsuccessful match" for cases with wrong comparison spectra). Validation and cross-validation results suggested that, for each peptide isomer, deconvolution was successful both in terms of the fragment ions present and their relative abundances in each mass spectrum.

\subsection{Arrival time correction factor and CCS calculations}

Although match scores (i.e., R values) for all deconvoluted CID mass spectra (supporting information, Figure S2) for the pentapeptides suggested that MS deconvolution was successful, the degree of match between ATs of deconvoluted and pure isomers could not be discerned or quantified from the MS data alone. In fact, as a result of the electric potential difference required to induce post-IM/CID, ATs extracted from the deconvoluted post-IM/CID data in Figure 1 did not match ATs of individually analyzed peptides (i.e., without post-IM/CID). The higher potential difference between the IM and transfer cells in post-IM/CID experiments results in faster acceleration of the ions as they exit the IM cell and pass through the transfer cell and thus 
leads to shorter measured ATs than what is obtained in low-energy (non-fragmentation) experiments. For example, the AT of deconvoluted (high-energy) $[\mathrm{SDGRG}+\mathrm{H}]^{+}(3.18 \pm 0.06$ ms: green IM profile in Figure 2a) was approximately $0.22 \mathrm{~ms}$ shorter than the AT for the individually analyzed (low-energy) pure $[\mathrm{SDGRG}+\mathrm{H}]^{+}(3.40 \pm 0.10 \mathrm{~ms}$ : green IM profile in Figure 2b); these AT values are statistically different at the 95\% C.L. and suggest that deconvoluted ATs should only be used for CCS calculations after applying an appropriate AT correction factor to the deconvoluted IM spectra.

One solution to avoid AT variations would be to run the CCS calibration mixture at the same post-IM/CID energy as used for deconvolution. However, in cases where the CCS calibrant is a polymer (such as poly-alanine used here) [42, 45, 63, 64], post-IM/CID could generate several isomers with different and/or overlapping IM profiles (e.g., CID of Alas through Ala $_{n}$ $\left\{\right.$ where $\mathrm{n}>5$ \} can generate $\mathrm{Ala}_{1-5}$ ), or completely fragment the precursor ion (e.g., Alas is depleted while CID of $\mathrm{Ala}_{\mathrm{n}}$ \{where $\left.\mathrm{n}>5\right\}$ still generates the Alas fragment ion), and complicate the extraction of pure IM ATs. Moreover, if post-IM/CID were used for a calibration mixture, then calibration plots would only be valid for that specific collision energy. Therefore, multiple calibration profiles would have to be generated to calculate CCS values for IM-unresolved species deconvoluted using different collision energies [46]. Hence, it would be advantageous to correct ATs of deconvoluted IM profiles and fit them to a "conventional" low-energy CCS calibration plot.

To correct for change in ATs caused by post-IM/CID acceleration voltage, we ran the pentapeptide mixture at low collision energy (i.e., $4 \mathrm{~V}$; which is insufficient to induce CID) and high collision energy (i.e., $40 \mathrm{~V}$ ) and calculated the difference in AT (herein referred to as the AT "shift factor"). We then added this shift factor (of $\sim 0.13 \mathrm{~ms}$ ) to ATs of deconvoluted IM 
profiles to generate corrected-IM distributions (Figure 2c, discussed in-detail below); note that similar shift factors can be applied to all ions exiting the IM cell. Garmón-Lobato et al. presented a similar strategy for aligning low- and high-energy IM profiles [65]; however, their approach was not focused on characterizing ions with similar CCS values, such as those presented in this manuscript, or calculating CCS values from the resulting aligned spectra [65].

\subsection{Estimation of Time Correction Factor}

The magnitude of AT correction can be estimated from known parameters of the experiment. Experimentally measured ATs in the Synapt G1 (and G2-S) instruments are the summation of four ion transit times including: (1) IM drift time, (2) time spent in the transfer cell, (3) TOF MS flight time, and (4) time spent traveling across the interface between each of these instrument regions $[11,28,66]$. IM drift times should not change significantly between low- and high-energy experiments because the electric fields (i.e., TW, entrance and exit lenses, and radio frequency confinement voltages) and IM pressure, which govern ion mobilities through the IM cell, are the same in both sets of experiments [28, 67]. Ion flight times within the TOF analyzer for precursor ions remain constant for both low-energy (no CID) and high-energy (post-IM/CID) experiments. However, due to larger potential difference between IM and transfer cells during a high-energy post-IM/CID experiment, molecular and potential fragment ions should spend less time in this interface region and exhibit mass-dependent time shifts.

In the Synapt G1, the center-to-center spacing between ring electrodes (e.g., between the IM and transfer cells) is $\sim 1.5 \mathrm{~mm}$ and the length of the transfer cell is $\sim 100 \mathrm{~mm}[66,67]$. Based on the physical geometry and electric fields of the instrument [66, 67], ions will spend a considerably longer time in the transfer cell than in the region between the IM and transfer cells or in the TOF mass spectrometer. As a result, we can approximate a maximum AT shift factor to 
be equal to the maximum amount of time an ion can spend in the transfer cell. The transfer cell is operated at lower pressure than the IM cell and ions therefore travel with the TW rather than falling over the wave as they might do in $\operatorname{IM}[14,21,66,67]$. Therefore, assuming an ion entering the transfer cell had insufficient kinetic energy to overtake the TW within the transfer cell, and ignoring phase effects of the TW, the maximum amount of time an ion could spend in the transfer cell ( $\left.t_{\text {transfer }}\right)$ would be equal to the length of the cell $\left(d_{t}\right)$ divided by the transfer cell wave velocity $\left(v_{t}\right)[11]$ :

\section{Equation 2$$
t_{\text {transfer }}=\frac{d_{t}}{v_{t}}
$$

For a cell length of $100 \mathrm{~mm}(0.1 \mathrm{~m})$ and transfer cell wave velocity of $248 \mathrm{~m} / \mathrm{s}$ (as used in our experiments), the maximum time an ion should spend in the transfer cell (under our experimental conditions) is $\sim 0.40 \mathrm{~ms}$. Based on these simple estimations, under our experimental conditions, we should expect shift factors of less than $\sim 0.40 \mathrm{~ms}$ (maximum transfer cell time).

\subsection{Empirical shift factors}

Figure 3 shows the observed shift in IM AT for the binary pentapeptide mixture at $4 \mathrm{~V}$ (black IM profile) and $40 \mathrm{~V}$ (blue IM profile) post-IM/CID collision-energies. The measured shift factor for 7.6 V IM wave height was consistently $0.13 \mathrm{~ms}$ across eight trials (i.e., for four low-energy (4 V) and four high-energy (40 V) experiments). As expected, calculated shift factors varied as a function of wave height, ranging from $0.12 \mathrm{~ms}$ to $0.18 \mathrm{~ms}$ for wave heights of $7.0 \mathrm{~V}$ to $8.0 \mathrm{~V}$ (specific values can be found in the supporting information, Spreadsheet S1). Experimentally determined AT shift factors (i.e., $0.12 \mathrm{~ms}$ to $0.18 \mathrm{~ms}$ ) are within the estimated range of less than $\sim 0.40 \mathrm{~ms}$ (i.e., as discussed in the preceding section). Deconvoluted IM profiles were corrected by adding the AT shift factor (i.e., $0.13 \mathrm{~ms}$ ) to the IM profiles in Figure 2a to yield time-corrected IM profiles (Figure 2c). ATs for individually measured peptide 
isomers and AT-corrected deconvoluted IM profiles at a wave height of $7.6 \mathrm{~V}$ were statistically indistinguishable at the $95 \%$ confidence level. Because of the approximations involved in the deconvolution process (e.g., peak centroid determination) [39] and mobility-dependent ion velocity, adding a single correction factor is not expected to improve all AT values identically. For example, the average corrected-AT value for GRGDS (3.52 ms, from the red IM profile in Figure $2 \mathrm{c})$ is closer to its individually measured value $(3.50 \mathrm{~ms}$, from the red IM profile in Figure 2b) than the corrected-AT for SDGRG (3.31 ms, from the green IM profile in Figure 2c) is to its individually measured value ( $3.40 \mathrm{~ms}$, from the green IM profile in Figure $2 b)$. However, based on the aforementioned statistical justifications, corrected-deconvoluted ATs (as labeled in Figure 2c) were sufficient for use in CCS calculations (i.e., CCS results from AT-corrected deconvolution and pure IM peak analyses agreed at the 95\% C.L.).

Table 1 contains a summary of CCS calculations for the pentapeptide mixture. The calculated CCS of the pentapeptide mixture (i.e., 210.3 $\pm 3.2 \AA^{2}$; where the subscript digit denotes the first insignificant figure) is comparable to the mathematical average CCS $\left(\sim 211 \AA^{2}\right)$ of individually measured $[\mathrm{SDGRG}+\mathrm{H}]^{+}\left(209.1 \pm 3.1 \AA^{2}\right)$ and $[\mathrm{GRGDS}+\mathrm{H}]^{+}\left(212.7 \pm 3.3 \AA^{2}\right)$ (Table 1). Our calculated CCS values for individual $[\mathrm{SDGRG}+\mathrm{H}]^{+}$and $[\mathrm{GRGDS}+\mathrm{H}]^{+}$(from pure samples) are within the $3 \%$ error range of the previously reported values for the same peptide samples calculated using DTIM-MS [68]. Slight differences in CCS measurements may be due to several factors including (but not limited to) differences in IM cell humidity [69, 70], mixture of gasses between the trap/transfer and IM cells (i.e., changing the reduced mass and average drift gas polarizability) [45, 68, 71-73], accuracy and precision of calibrant CCS values [68], and/or other experimental condition variations [10]. CCS values of deconvoluted [SDGRG $+\mathrm{H}]^{+}$and $[\mathrm{GRGDS}+\mathrm{H}]^{+}$without AT corrections were $204.9 \pm 3.0 \AA^{2}$ and $209.5 \pm 3.1 \AA^{2}$, 
respectively (Table 1). Uncorrected CCS values were not statistically different than the individually measured values for both peptides at the $95 \%$ confidence level; however, at the $90 \%$ C.L., the uncorrected CCS for $[\mathrm{SDGRG}+\mathrm{H}]^{+}$was significantly different than the corresponding pure CCS. By comparison, calculated CCS values for the AT-corrected deconvoluted IM profiles for $[\mathrm{SDGRG}+\mathrm{H}]^{+}$and $[\mathrm{GRGDS}+\mathrm{H}]^{+}$were $208.3 \pm 4.4 \AA^{2}$ and $212.7 \pm 4.6 \AA^{2}$, respectively (Table 1); these calculated CCS values (after applying the AT correction approach) are not statistically different than the CCS values of individually measured peptide isomers at either the $95 \%$ or $90 \%$ confidence levels.

Our results indicate that CCS values of IM-unresolved reverse sequence peptide isomers (i.e., SDGRG and GRGDS) can be accurately calculated using chemometric deconvolution and AT correction using a Synapt G1 system. In the next section, we extend those results to demonstrate the utility of IM-unresolved CCS calculations on a four-component IM-unresolved enkephalin isomer mixture (i.e., RYGGFM, RMFGYG, MFRYGG, and FRMYGG) using a higher IM resolving power Synapt G2-S system.

\subsection{Application of IM-unresolved CCS calculations using a Synapt G2-S system}

Clemmer and coworkers demonstrated that by using LC-IM-MS they could identify $\sim 82 \%$ of isomers in an approximately 4000-component combinatorial peptide library [74]. Thus, although this study demonstrated that the majority of isomers in a complex mixture could be differentiated with LC and IM, there were still isomers that remained unresolved. In order to test our approach for a "worst case scenario" we analyzed a mixture of four constitutional isomeric enkephalin hexapeptides; to the best our knowledge, CCS values for these isomers have not been reported. Results from peak deconvolution in TWIM-MS experiments were compared to 
calculated theoretical CCS values (i.e., using PSA) and experimentally derived DTIM-MS values.

Figure 4 shows the IM profile for a doubly-protonated $(m / z, 365.67)$ four-component hexapeptide mixture before (black IM profile) and after (colored IM profiles where the orange, purple, cyan, and gray IM profiles correspond to RYGGFM, RMFGYG, MFRYGG, and FRMYGG, respectively) IM deconvolution at IM wave height of $22 \mathrm{~V}$ and wave velocity of $1300 \mathrm{~m} / \mathrm{s}$. The enkephalin isomer mixture (Figure 4, black IM profile) yielded a bimodal IM distribution, which could erroneously be characterized as corresponding to two components if peak widths of the two overlapping Gaussian-like distributions were not considered. For example, the peak width of the later AT profile (which is generated from the additive sum of three individual IM profiles, as discussed in detail below) is much broader than the early AT conformer (which corresponds to a single isomer) [75].

Deconvolution, $R$ and $R^{\prime}$ match scoring, and AT corrections were performed as discussed in the preceding sections. Calculated $R$ and $R^{\prime}$ values for all deconvoluted CID mass spectra for the hexapeptides were $\geq 0.83$ and $\leq 0.37$, respectively. For brevity, only final CCS values for the hexapeptides are discussed in this section. Examples of deconvoluted and pure CID mass spectra for hexapeptide isomers can be found in the supporting information (Figure S1).

Table 2 shows a summary of CCS calculations for the enkephalin isomer mixture. Similar to the pentapeptide mixture (Table 1), if the late-AT IM profile in Figure 4 was misidentified as a single component, then the calculated CCS (i.e., 296.9 $\pm 7.0 \AA^{2}$ ) would be close to the average of the CCS values of the individual IM-unresolved isomers that contribute to the late-AT IM peak. Likewise, the calculated CCS for the early-AT profile in the hexapeptide mixture (i.e., without 
deconvolution; $288.8 \pm 6.8 \AA^{2}$ ) at the 95\% C.L. is statistically indistinguishable from the CCS of the first eluting hexapeptide (i.e., $[\mathrm{RYGGFM}+2 \mathrm{H}]^{2+} ; 292.7 \pm 6.9 \AA^{2}$ ).

Collision cross sections calculated for the AT-corrected deconvoluted IM profiles were statistically indistinguishable from the corresponding individually analyzed peptides (Table 2 and supporting information, Spreadsheet S2). Although results for neither raw nor AT-corrected deconvoluted IM data were statistically different from the results obtained for the individual peptides, average CCS values for the corrected-AT deconvolution results were closer to the average CCS values for the individually analyzed peptides. For example, the calculated CCS for individually measured $[\mathrm{FRMYGG}+2 \mathrm{H}]^{2+}$ was $301.5 \pm 7.0 \AA^{2}$, which is closer to the calculated CCS for the AT-corrected deconvoluted data of 300.1 $\pm 10.1 \AA^{2}$ than to the estimated CCS value of $297.1 \pm 7.1 \AA^{2}$ for deconvoluted raw data (prior to AT-correction). These results demonstrate that AT-correction improves CCS calculations (both for data from Synapt G1 and G2-S instruments) and can be applied to data from complex mixtures containing more than two IMoverlapping components.

To further demonstrate the accuracy of CCS values calculated from AT-corrected deconvoluted IM profiles, we analyzed the same enkephalin peptides on a DTIM-MS and experimentally measured their individual CCS values. Results from DTIM-MS measurements are summarized in Table 2. As listed in Table 2, CCS values calculated from TW and DT type instruments are in close agreement, with less than $\sim 1.3 \%$ deviation from DT values in the worst case. Moreover, CCS values derived from AT-corrected post-TWIM/CID MS deconvoluted data are not significantly different than CCS values derived from the individually analyzed peptides analyzed on the DTIM-MS instrument (i.e., agree at the 95\% confidence level; see Spreadsheet S2). 
The number of significant figures in CCS values calculated using TWIM-MS (Tables 1 and 2) were limited by the number of significant figures (viz. accuracy) reported for calibrant CCS literature values. Therefore, TWIM-MS CCS values are reported to three significant figures whereas DTIM-MS CCS values (which are not limited by the calibrant CCS values) were reported to four significant figures. All experimental errors reported in this manuscript were calculated by propagating error through each step of CCS calculations. For DTIM-MS measurements, the primary error is in the recorded ATs and in the AT correction (i.e., to compensate for time outside of the IM portion of the instrument; see Spreadsheet S3). For TWIM-MS measurements, there are errors associated with calibrant and analyte ATs (and error in "shift factors" used for AT-correction), fitting parameters, and exponential calibration factors derived from calibrant ATs. TWIM-MS calibration errors were calculated using the linear least squares fit and errors (LINEST) function in Microsoft Excel (see Spreadsheets S1 and S2). Although error propagation calculations can be time consuming, they more accurately represent the precision of IM measurements and underscore the importance of developing a calibration independent TWIM-MS CCS equation. Nevertheless, our results suggest that CCS values for IM-unresolved isomers can be calculated using deconvolution of post-IM/CID MS data; moreover, statistical comparisons of CCS values calculated using deconvoluted TWIM-MS data and directly measured DTIM-MS data indicate that these values are indistinguishable.

In addition to the DT CCS values obtained for the hexapeptide isomers, theoretical CCS values were also determined for an ensemble of conformations resulting from molecular dynamics simulations. Good agreement is observed between experimentally measured (ATcorrected deconvoluted TWIM-MS) CCS values (vertical lines) and the conformational space plots (calculated using PSA with a preliminary parameter set for nitrogen) in Figure 5. Root- 
mean-squared deviation (RMSD) clustering representative conformations obtained from each of the isomers that fall within the experimental range (i.e., experimentally determined CCS $\pm 95 \%$ C.L.) are also shown in Figure 5 [61]. The structure of $[\mathrm{RYGGFM}+2 \mathrm{H}]^{2+}$, with the two glycine amino acids located at the center of the peptide chain (Figure 5a), is expected to have increased flexibility in the gas-phase and corresponds to the peptide ion with the lowest CCS among its four isomers in Table 2. The other three isomers have similar CCS values (as confirmed by their

overlapping IM traces shown in Figure 4). The peptide ion, $[\mathrm{RMFGYG}+2 \mathrm{H}]^{2+}$ (Figure 5b) with one internal glycine is predicted to have some flexibility and this ion possesses the next largest CCS of the series. On the other hand, the N-terminal glycines present in both $[\mathrm{MFRYGG}+2 \mathrm{H}]^{2+}$ and $[\text { FRMYGG }+2 \mathrm{H}]^{2+}$ (Figures $5 \mathrm{c}$ and $5 \mathrm{~d}$ ) presumably restrict the flexibility of these ions in the gas-phase. Interaction of the positive arginine residue with the $\mathrm{C}$-terminus carboxylate group in Figure $5 \mathrm{~d}$ is expected to extend the structure by pushing out the tryptophan and phenylalanine benzene rings of $[\mathrm{FRMYGG}+2 \mathrm{H}]^{2+}$ which we interpret to contribute to the largest CCS among the four isomers. Additional RMSD clustered representative conformations corresponding to the experimental range of the four isomers are provided in the supplemental materials (Figures S3S6).

\section{Conclusion}

We presented a method to calculate the CCS values of IM-unresolved isomers from TWIM-MS measurements using a modified version of the calibration protocol proposed by Ruotolo et al. [11]. To accurately measure CCS values for unresolved isomers, it was necessary to calculate IM AT "shift factors" which are used to correct the ATs of chemometrically deconvoluted IM profiles. Use of these shift factors improved the measured CCS values as compared to CCS values obtained from pure analytical standards analyzed on both TWIM-MS 
and DTIM-MS instruments. Moreover, the CCS values calculated using IM-deconvoluted data matched closely with theoretical conformational space scatter plots constructed using PSA.

Currently, CCS calibration for TWIM-MS yields measured CCS values with larger errors (e.g., $\sim 2 \%$ to $3 \%$ of the mean CCS values, see Tables 1 and 2$)$ than comparable DTIM-MS values (e.g., $<1 \%$ of the mean CCS values, see Table 2). Because of these large experimental errors (relative to DTIM-MS measurements), CCS values of some AT non-corrected deconvoluted data were not statistically different than individually measured values (see discussion of data presented in Table 1 and 2 above). However, agreements between the individually measured CCS values and deconvoluted CCS values improves after applying AT correction (see Tables 1 and 2). As more precise calibration and instrumental techniques are developed, CCS measurement errors should (ideally) decrease. Similarly, as intra-instrument errors decrease, the use of AT-correction for post-IM acceleration will become more important for calculating more accurate CCS values.

In this report, we utilized TWIM-MS instruments for convolution experiments; however, the proposed time-corrected deconvolution technique is not limited to TWIM-MS and AT shift factors can be calculated for any IM-MS instrument capable of post-IM/CID. Moreover, the reported protocol could also be utilized in other MS techniques that exploit post-IM/CID[33] to determine the presence of unresolved species and extract CCS information from subsequently deconvoluted spectra.

\section{Acknowledgements}

We thank National Science Foundation (NSF-IDBR, award number 1455668) and Baylor University for financial support. Support for Vanderbilt authors was provided by the National Institutes of Health (Grants 2R01GM092218, 5UH3TR000491-04, and 3UH3TR000491-0451), and the National Science Foundation (CHE-1229341). We also thank Matthew Brantley for 
assistance with calculating CID mass spectra matching scores and Brooke Brown for performing limited experimental replicates.

\section{References}

[1] Cumeras, R., Figueras, E., Davis, C. E., Baumbach, J. I., Gracia, I., Review on Ion Mobility Spectrometry. Part 1: current instrumentation, Analyst 140 (2015) 1376-1390.

[2] May, J. C., McLean, J. A., Ion Mobility-Mass Spectrometry: Time-Dispersive Instrumentation, Anal. Chem. 87 (2015) 1422-1436.

[3] Ruotolo, B. T., Giles, K., Campuzano, I., Sandercock, A. M., Bateman, R. H., Robinson, C. V., Evidence for Macromolecular Protein Rings in the Absence of Bulk Water, Science 310 (2005) 1658-1661.

[4] Scarff, C. A., Thalassinos, K., Hilton, G. R., Scrivens, J. H., Travelling wave ion mobility mass spectrometry studies of protein structure: biological significance and comparison with $\mathrm{X}$ ray crystallography and nuclear magnetic resonance spectroscopy measurements, Rapid Commun. Mass Spectrom. 22 (2008) 3297-3304.

[5] Wyttenbach, T., Bowers, M. T., Structural Stability from Solution to the Gas Phase: Native Solution Structure of Ubiquitin Survives Analysis in a Solvent-Free Ion Mobility-Mass Spectrometry Environment, J. Phys. Chem. B 115 (2011) 12266-12275.

[6] Bleiholder, C., Dupuis, N. F., Wyttenbach, T., Bowers, M. T., Ion mobility-mass spectrometry reveals a conformational conversion from random assembly to $\beta$-sheet in amyloid fibril formation, Nat. Chem. 3 (2011) 172-177.

[7] Zhong, Y., Hyung, S.-J., Ruotolo, B. T., Ion mobility-mass spectrometry for structural proteomics, Expert Rev. Proteomics 9 (2012) 47-58.

[8] Jurneczko, E., Barran, P. E., How useful is ion mobility mass spectrometry for structural biology? The relationship between protein crystal structures and their collision cross sections in the gas phase, Analyst 136 (2011) 20-28.

[9] Chen, S.-H., Russell, D., How Closely Related Are Conformations of Protein Ions Sampled by IM-MS to Native Solution Structures?, J. Am. Soc. Mass Spectrom. 26 (2015) 1433-1443.

[10] Cumeras, R., Figueras, E., Davis, C., Baumbach, J., Gràcia, I., Review on Ion Mobility Spectrometry. Part 2: hyphenated methods and effects of experimental parameters, Analyst 140 (2015) 1391-1410.

[11] Ruotolo, B. T., Benesch, J. L. P., Sandercock, A. M., Hyung, S.-J., Robinson, C. V., Ion mobility-mass spectrometry analysis of large protein complexes, Nat. Prot. 3 (2008) 1139-1152.

[12] Rokushika, S., Hatano, H., Baim, M. A., Hill, H. H., Resolution measurement for ion mobility spectrometry, Anal. Chem. 57 (1985) 1902-1907.

[13] Siems, W. F., Wu, C., Tarver, E. E., Hill, H. H., Jr., Larsen, P. R., McMinn, D. G., Measuring the Resolving Power of Ion Mobility Spectrometers, Anal. Chem. 66 (1994) 41954201.

[14] Shvartsburg, A. A., Smith, R. D., Fundamentals of Traveling Wave Ion Mobility Spectrometry, Anal. Chem. 80 (2008) 9689-9699.

[15] May, J. C., Dodds, J. N., Kurulugama, R. T., Stafford, G. C., Fjeldsted, J. C., McLean, J. A., Broadscale resolving power performance of a high precision uniform field ion mobility-mass spectrometer, Analyst 140 (2015) 6824-6833. 
[16] Dwivedi, P., Wu, C., Matz, L. M., Clowers, B. H., Siems, W. F., Hill, H. H., Gas-Phase Chiral Separations by Ion Mobility Spectrometry, Anal. Chem. 78 (2006) 8200-8206.

[17] Shvartsburg, A. A., Smith, R. D., Accelerated High-Resolution Differential Ion Mobility Separations Using Hydrogen, Anal. Chem. 83 (2011) 9159-9166.

[18] Fasciotti, M., Sanvido, G. B., Santos, V. G., Lalli, P. M., McCullagh, M., de Sá, G. F., Daroda, R. J., Peter, M. G., Eberlin, M. N., Separation of isomeric disaccharides by traveling wave ion mobility mass spectrometry using CO2 as drift gas, J. Mass Spectrom. 47 (2012) 16431647.

[19] Liang, X., Wang, X., Wang, W., Zhou, Q., Chen, C., Peng, L., Wen, M., Qu, T., Wang, Z., Zhao, K., Li, J., Li, H., Sensitive detection of black powder by stand-alone ion mobility spectrometer with chlorinated hydrocarbon modifiers in drift gas, Talanta 121 (2014) 215-219.

[20] Purves, R. W., Ozog, A. R., Ambrose, S. J., Prasad, S., Belford, M., Dunyach, J.-J., Using gas modifiers to significantly improve sensitivity and selectivity in a cylindrical FAIMS device, J. Am. Soc. Mass Spectrom. 25 (2014) 1274-1284.

[21] May, J., McLean, J., The influence of drift gas composition on the separation mechanism in traveling wave ion mobility spectrometry: insight from electrodynamic simulations, Int. J. Ion Mobil. Spectrom. 16 (2013) 85-94.

[22] Liu, Y., Clemmer, D. E., Characterizing Oligosaccharides Using Injected-Ion Mobility/Mass Spectrometry, Anal. Chem. 69 (1997) 2504-2509.

[23] Hoaglund-Hyzer, C. S., Li, J., Clemmer, D. E., Mobility Labeling for Parallel CID of Ion Mixtures, Anal. Chem. 72 (2000) 2737-2740.

[24] Clowers, B., Dwivedi, P., Steiner, W., Hill, H., Bendiak, B., Separation of sodiated isobaric disaccharides and trisaccharides using electrospray ionization-atmospheric pressure ion mobilitytime of flight mass spectrometry, J. Am. Soc. Mass Spectrom. 16 (2005) 660-669.

[25] Clowers, B. H., Ibrahim, Y. M., Prior, D. C., Danielson, W. F., Belov, M. E., Smith, R. D., Enhanced Ion Utilization Efficiency Using an Electrodynamic Ion Funnel Trap as an Injection Mechanism for Ion Mobility Spectrometry, Anal. Chem. 80 (2008) 612-623.

[26] Zucker, S., Lee, S., Webber, N., Valentine, S., Reilly, J., Clemmer, D., An Ion Mobility/Ion Trap/Photodissociation Instrument for Characterization of Ion Structure, J. Am. Soc. Mass Spectrom. 22 (2011) 1477-1485.

[27] Hernandez, D. R., DeBord, J. D., Ridgeway, M. E., Kaplan, D. A., Park, M. A., FernandezLima, F., Ion dynamics in a trapped ion mobility spectrometer, Analyst 139 (2014) 1913-1921.

[28] Giles, K., Williams, J. P., Campuzano, I., Enhancements in travelling wave ion mobility resolution, Rapid Commun. Mass Spectrom. 25 (2011) 1559-1566.

[29] Xuan, Y., Creese, A. J., Horner, J. A., Cooper, H. J., High-field asymmetric waveform ion mobility spectrometry (FAIMS) coupled with high-resolution electron transfer dissociation mass spectrometry for the analysis of isobaric phosphopeptides, Rapid Commun. Mass Spectrom. 23 (2009) 1963-1969.

[30] Creese, A. J., Cooper, H. J., Separation and Identification of Isomeric Glycopeptides by High Field Asymmetric Waveform Ion Mobility Spectrometry, Anal. Chem. 84 (2012) 25972601.

[31] Lee, S., Li, Z., Valentine, S. J., Zucker, S. M., Webber, N., Reilly, J. P., Clemmer, D. E., Extracted fragment ion mobility distributions: A new method for complex mixture analysis, Int. J. Mass Spectrom. 309 (2012) 154-160.

[32] Zhu, F., Lee, S., Valentine, S., Reilly, J., Clemmer, D., Mannose7 Glycan Isomer Characterization by IMS-MS/MS Analysis, J. Am. Soc. Mass Spectrom. 23 (2012) 2158-2166. 
[33] Hoffmann, W., Hofmann, J., Pagel, K., Energy-Resolved Ion Mobility-Mass Spectrometry-A Concept to Improve the Separation of Isomeric Carbohydrates, J. Am. Soc. Mass Spectrom. 25 (2014) 471-479.

[34] Khakinejad, M., Kondalaji, S., Maleki, H., Arndt, J., Donohoe, G., Valentine, S., Combining Ion Mobility Spectrometry with Hydrogen-Deuterium Exchange and Top-Down MS for Peptide Ion Structure Analysis, J. Am. Soc. Mass Spectrom. 25 (2014) 2103-2115.

[35] Harvey, D., Crispin, M., Bonomelli, C., Scrivens, J., Ion Mobility Mass Spectrometry for Ion Recovery and Clean-Up of MS and MS/MS Spectra Obtained from Low Abundance Viral Samples, J. Am. Soc. Mass Spectrom. (2015) 1-14.

[36] Colby, B. N., Spectral deconvolution for overlapping GC/MS components, J. Am. Soc. Mass Spectrom. 3 (1992) 558-562.

[37] Zekavat, B., Solouki, T., Chemometric Data Analysis for Deconvolution of Overlapped Ion Mobility Profiles, J. Am. Soc. Mass Spectrom. 23 (2012) 1873-1884.

[38] Windig, W., Guilment, J., Interactive self-modeling mixture analysis, Anal. Chem. 63 (1991) 1425-1432.

[39] Brantley, M., Zekavat, B., Harper, B., Mason, R., Solouki, T., Automated Deconvolution of Overlapped Ion Mobility Profiles, J. Am. Soc. Mass Spectrom. 25 (2014) 1810-1819.

[40] Meloun, M., Čapek, J., Mikšík, P., Brereton, R. G., Critical comparison of methods predicting the number of components in spectroscopic data, Anal. Chim. Acta 423 (2000) 51-68.

[41] Malinowski, E. R., Determination of the number of factors and the experimental error in a data matrix, Anal. Chem. 49 (1977) 612-617.

[42] Zekavat, B., Miladi, M., Becker, C., Munisamy, S., Solouki, T., Combined Use of Post-Ion Mobility/Collision-Induced Dissociation and Chemometrics for b Fragment Ion Analysis, J. Am. Soc. Mass Spectrom. 24 (2013) 1355-1365.

[43] Zekavat, B., Miladi, M., Al-Fdeilat, A., Somogyi, A., Solouki, T., Evidence for Sequence Scrambling and Divergent H/D Exchange Reactions of Doubly-Charged Isobaric b-Type Fragment Ions, J. Am. Soc. Mass Spectrom. 25 (2014) 226-236.

[44] Harper, B., Miladi, M., Solouki, T., Loss of Internal Backbone Carbonyls: Additional Evidence for Sequence-Scrambling in Collision-Induced Dissociation of y-Type Ions, J. Am. Soc. Mass Spectrom. 25 (2014) 1716-1729.

[45] Bush, M. F., Campuzano, I. D. G., Robinson, C. V., Ion Mobility Mass Spectrometry of Peptide Ions: Effects of Drift Gas and Calibration Strategies, Anal. Chem. 84 (2012) 7124-7130. [46] Pettit, M. E., Harper, B., Brantley, M. R., Solouki, T., Collision-energy resolved ion mobility characterization of isomeric mixtures, Analyst 140 (2015) 6886-6896.

[47] May, J. C., Goodwin, C. R., Lareau, N. M., Leaptrot, K. L., Morris, C. B., Kurulugama, R. T., Mordehai, A., Klein, C., Barry, W., Darland, E., Overney, G., Imatani, K., Stafford, G. C., Fjeldsted, J. C., McLean, J. A., Conformational Ordering of Biomolecules in the Gas Phase: Nitrogen Collision Cross Sections Measured on a Prototype High Resolution Drift Tube Ion Mobility-Mass Spectrometer, Anal. Chem. 86 (2014) 2107-2116.

[48] May, J. C., McLean, J. A., A Uniform Field Ion Mobility Study of Melittin and Implications of Low-Field Mobility for Resolving Fine Cross-Sectional Detail in Peptide and Protein Experiments, Proteomics (2015).

[49] Mason, E. A., Schamp Jr, H. W., Mobility of gaseous lons in weak electric fields, Ann. Phys. 4 (1958) 233-270.

[50] Case, D. A., Babin, V., Berryman, J., Betz, R. M., Cai, Q., Cerutti, D. S., Cheatham Iii, T. E., Darden, T. A., Duke, R. E., Gohlke, H., Amber 14, (2014). 
[51] Frisch, M. J., Trucks, G. W., Schlegel, H. B., Scuseria, G. E., Robb, M. A., Cheeseman, J. R., Scalmani, G., Barone, V., Mennucci, B., Petersson, G. A., Gaussian 09, Revision A. 02, Gaussian, Inc., Wallingford, CT 2 (2009) 4.

[52] Forsythe, J. G., Stow, S. M., Nefzger, H., Kwiecien, N. W., May, J. C., McLean, J. A., Hercules, D. M., Structural Characterization of Methylenedianiline Regioisomers by Ion Mobility-Mass Spectrometry, Tandem Mass Spectrometry, and Computational Strategies: I. Electrospray Spectra of 2-Ring Isomers, Anal. Chem. 86 (2014) 4362-4370.

[53] Stow, S. M., Onifer, T. M., Forsythe, J. G., Nefzger, H., Kwiecien, N. W., May, J. C., McLean, J. A., Hercules, D. M., Structural Characterization of Methylenedianiline Regioisomers by Ion Mobility-Mass Spectrometry, Tandem Mass Spectrometry, and Computational Strategies. 2. Electrospray Spectra of 3-Ring and 4-Ring Isomers, Anal. Chem. 87 (2015) 6288-6296.

[54] von Helden, G., Hsu, M. T., Gotts, N., Bowers, M. T., Carbon cluster cations with up to 84 atoms: structures, formation mechanism, and reactivity, J. Phys. Chem. 97 (1993) 8182-8192.

[55] Mesleh, M. F., Hunter, J. M., Shvartsburg, A. A., Schatz, G. C., Jarrold, M. F., Structural information from ion mobility measurements: effects of the long-range potential, J. Phys. Chem. 100 (1996) 16082-16086.

[56] Shvartsburg, A. A., Jarrold, M. F., An exact hard-spheres scattering model for the mobilities of polyatomic ions, Chem. Phys. Lett. 261 (1996) 86-91.

[57] Campuzano, I., Bush, M. F., Robinson, C. V., Beaumont, C., Richardson, K., Kim, H., Kim, H. I., Structural Characterization of Drug-like Compounds by Ion Mobility Mass Spectrometry: Comparison of Theoretical and Experimentally Derived Nitrogen Collision Cross Sections, Anal. Chem. 84 (2012) 1026-1033.

[58] Bleiholder, C., Wyttenbach, T., Bowers, M. T., A novel projection approximation algorithm for the fast and accurate computation of molecular collision cross sections (I). Method, Int. J. Mass Spectrom. 308 (2011) 1-10.

[59] Bleiholder, C., Contreras, S., Do, T. D., Bowers, M. T., A novel projection approximation algorithm for the fast and accurate computation of molecular collision cross sections (II). Model parameterization and definition of empirical shape factors for proteins, Int. J. Mass Spectrom. 345 (2013) 89-96.

[60] Anderson, S. E., Bleiholder, C., Brocker, E. R., Stang, P. J., Bowers, M. T., A novel projection approximation algorithm for the fast and accurate computation of molecular collision cross sections (III): Application to supramolecular coordination-driven assemblies with complex shapes, Int. J. Mass Spectrom. 330-332 (2012) 78-84.

[61] Bleiholder, C., Contreras, S., Bowers, M. T., A novel projection approximation algorithm for the fast and accurate computation of molecular collision cross sections (IV). Application to polypeptides, Int. J. Mass Spectrom. 354 (2013) 275-280.

[62] http://luschka.bic.ucsb.edu:8080/WebPSA/index.jsp

[63] Gidden, J., Wyttenbach, T., Jackson, A. T., Scrivens, J. H., Bowers, M. T., Gas-Phase Conformations of Synthetic Polymers: Poly(ethylene glycol), Poly(propylene glycol), and Poly(tetramethylene glycol), J. Am. Chem. Soc. 122 (2000) 4692-4699.

[64] Forsythe, J. G., Petrov, A. S., Walker, C. A., Allen, S. J., Pellissier, J. S., Bush, M. F., Hud, N. V., Fernandez, F. M., Collision cross section calibrants for negative ion mode traveling wave ion mobility-mass spectrometry, Analyst 140 (2015) 6853-6861.

[65] Garmón-Lobato, S., Abad-García, B., Sánchez-Ilárduya, M. B., Romera-Fernández, M., Berrueta, L. A., Gallo, B., Vicente, F., Improvement using chemometrics in ion mobility coupled 
to mass spectrometry as a tool for mass spectrometry fragmentation studies: Flavonoid aglycone cases, Anal. Chim. Acta 771 (2013) 56-64.

[66] Pringle, S. D., Giles, K., Wildgoose, J. L., Williams, J. P., Slade, S. E., Thalassinos, K., Bateman, R. H., Bowers, M. T., Scrivens, J. H., An investigation of the mobility separation of some peptide and protein ions using a new hybrid quadrupole/travelling wave IMS/oa-ToF instrument, Int. J. Mass Spectrom. 261 (2007) 1-12.

[67] Giles, K., Pringle, S. D., Worthington, K. R., Little, D., Wildgoose, J. L., Bateman, R. H., Applications of a travelling wave-based radio-frequency-only stacked ring ion guide, Rapid Commun. Mass Spectrom. 18 (2004) 2401-2414.

[68] Bush, M. F., Hall, Z., Giles, K., Hoyes, J., Robinson, C. V., Ruotolo, B. T., Collision cross sections of proteins and their complexes: a calibration framework and database for gas-phase structural biology, Anal. Chem. 82 (2010) 9557-9565.

[69] Mäkinen, M., Sillanpää, M., Viitanen, A. K., Knap, A., Mäkelä, J. M., Puton, J., The effect of humidity on sensitivity of amine detection in ion mobility spectrometry, Talanta 84 (2011) 116-121.

[70] Mayer, T., Borsdorf, H., Accuracy of Ion Mobility Measurements Dependent on the Influence of Humidity, Anal. Chem. 86 (2014) 5069-5076.

[71] Karpas, Z., Berant, Z., Effect of drift gas on mobility of ions, J. Phys. Chem. 93 (1989) 3021-3025.

[72] Asbury, G. R., Hill, H. H., Using Different Drift Gases To Change Separation Factors $(\alpha)$ in Ion Mobility Spectrometry, Anal. Chem. 72 (2000) 580-584.

[73] Beegle, L. W., Kanik, I., Matz, L., Hill Jr, H. H., Effects of drift-gas polarizability on glycine peptides in ion mobility spectrometry, Int. J. Mass Spectrom. 216 (2002) 257-268.

[74] Srebalus Barnes, C. A., Hilderbrand, A. E., Valentine, S. J., Clemmer, D. E., Resolving Isomeric Peptide Mixtures: A Combined HPLC/Ion Mobility-TOFMS Analysis of a 4000Component Combinatorial Library, Anal. Chem. 74 (2002) 26-36.

[75] Miladi, M., Zekavat, B., Munisamy, S. M., Solouki, T., A Systematic Study on the Effect of Histidine Position and Fragment Ion Size on the Formation of $\mathrm{b}_{n}$ Ions, Int. J. Mass Spectrom. 316-318 (2012) 164-173. 
Table 1. Experimental CCS Values for Two Reverse Pentapeptide Isomers

\begin{tabular}{ccccc}
\hline Identity & $\begin{array}{c}\text { Mixture } \\
\mathbf{C C S}\left(\AA^{2}\right)^{*}\end{array}$ & $\begin{array}{c}\text { Pure } \\
\mathbf{C C S}\left(\AA^{\mathbf{2}}\right)^{*}\end{array}$ & $\begin{array}{c}\text { Decon. } \\
\mathbf{C C S}\left(\AA^{\mathbf{2}}\right)^{*}\end{array}$ & $\begin{array}{c}\text { Corrected Decon. } \\
\mathbf{C C S}\left(\AA^{\mathbf{2}}\right)^{*}\end{array}$ \\
\hline $\begin{array}{c}{[\text { Reverse Peptides }+\mathrm{H}]^{+}} \\
\text {(mixture) }\end{array}$ & $210.3 \pm 3.2$ & - & - & - \\
{$[\text { GRGDS }+\mathrm{H}]^{+}$} & - & $212.7 \pm 3.3$ & $209.5 \pm 3.1$ & $212.7 \pm 4.6$ \\
{$[\text { SDGRG }+\mathrm{H}]^{+}$} & - & $209.1 \pm 3.1$ & $204.9 \pm 3.0$ & $208.3 \pm 4.4$ \\
\hline
\end{tabular}

*Subscript digits denote the first insignificant figure. Errors are reported at the 95\% C.L.

$\left(\mathrm{n}_{\text {mixtures }}=24, \mathrm{n}_{\text {pure }}=25\right)$.

Table 2. Experimental CCS Values for Four Enkephalin Hexapeptide Isomers

\begin{tabular}{|c|c|c|c|c|c|}
\hline Identity & $\begin{array}{c}\text { Mixture } \\
\operatorname{CCS}\left(\AA^{2}\right)^{*}\end{array}$ & $\begin{array}{c}\text { Pure } \\
\operatorname{CCS}\left(\AA^{2}\right)^{*}\end{array}$ & $\begin{array}{c}\text { Decon. } \\
\operatorname{CCS}\left(\AA^{2}\right)^{*}\end{array}$ & $\begin{array}{c}\text { Corrected Decon. } \\
\operatorname{CCS}\left(\AA^{2}\right)^{*}\end{array}$ & $\begin{array}{l}\text { Drift Tube } \\
\text { CCS }\left(\AA^{2}\right)^{*}\end{array}$ \\
\hline $\begin{array}{c}{[\text { Enkephalins }+2 \mathrm{H}]^{2+}} \\
\text { (mixture; early AT Peak) }\end{array}$ & $288.8 \pm 6.8$ & - & - & - & - \\
\hline $\begin{array}{c}{[\text { Enkephalins }+2 \mathrm{H}]^{2+}} \\
\text { (mixture; late AT Peak) }\end{array}$ & $296.9 \pm 7.0$ & - & - & - & - \\
\hline$[\mathrm{RYGGFM}+2 \mathrm{H}]^{2+}$ & - & $292.7 \pm 6.9$ & $285_{.7} \pm 6.7$ & $288.8 \pm 9.6$ & $285.2_{3} \pm 0.5_{3}$ \\
\hline$[\mathrm{RMFGYG}+2 \mathrm{H}]^{2+}$ & - & $299 \cdot 6 \pm 7 \cdot \cdot_{1}$ & $292.0 \pm 6.9$ & $295.1 \pm 9.9$ & $296.1_{1} \pm 0.3_{8}$ \\
\hline$[\mathrm{MFRYGG}+2 \mathrm{H}]^{2+}$ & - & $299.2 \pm 7.1$ & $293.8 \pm 6.9$ & $296.8 \pm 10_{.0}$ & $298.9_{7} \pm 0.4_{8}$ \\
\hline$[\mathrm{FRMYGG}+2 \mathrm{H}]^{2+}$ & - & $301.5 \pm 7.0$ & $297.1 \pm 7.1$ & $300_{\cdot 1} \pm 10_{\cdot 1}$ & $303.7_{5} \pm 0.4_{0}$ \\
\hline
\end{tabular}

*Subscript digits denote the first insignificant figure. Errors are reported at the 95\% C.L. (TWIMS: $\mathrm{n}_{\text {mixtures }}=$ $24, \mathrm{n}_{\text {pure }}=23$ (FRMYGG) or $\mathrm{n}_{\text {pure }}=24$ (all others); DTIMS: $\mathrm{n}=24$ (for each peptide) $)$ 


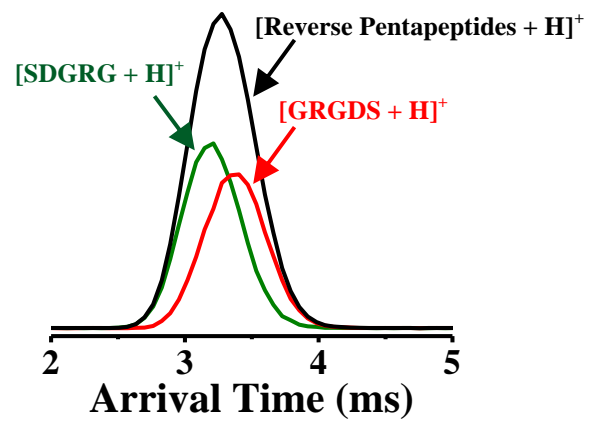

Figure 1. IM profiles of a binary mixture containing $[\mathrm{SDGRG}+\mathrm{H}]^{+}$(green) and $[\text {GRGDS }+\mathrm{H}]^{+}$ (red) before (black IM profile) and after (colored IM profiles) IM deconvolution using IM wave height voltage, wave velocity, and post-IM/CID acceleration voltage of $7.6 \mathrm{~V}, 300 \mathrm{~m} / \mathrm{s}$, and 40 $\mathrm{V}$, respectively.
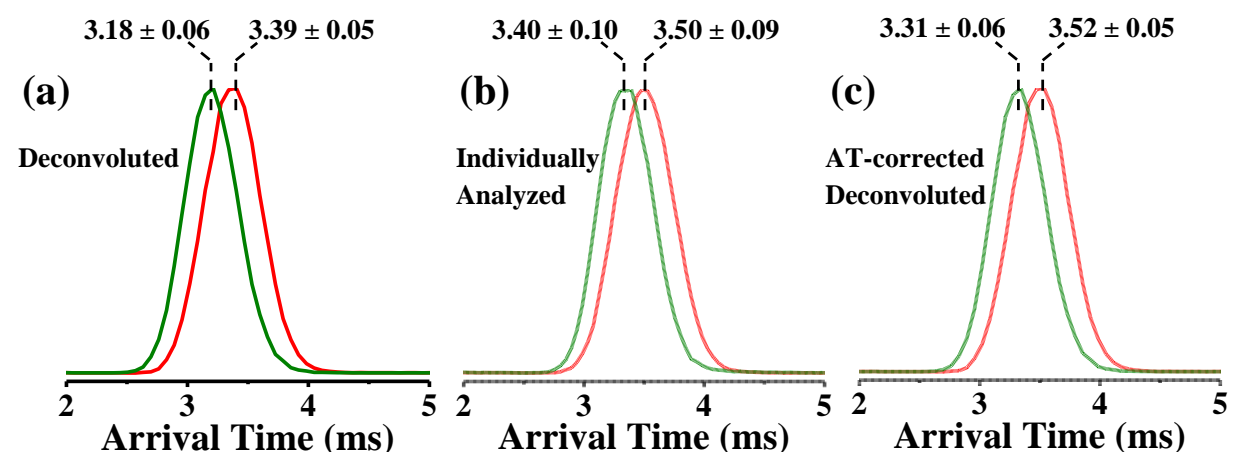

Figure 2. Normalized IM profiles for (a) deconvoluted, (b) individually analyzed pure, and (c) AT-corrected deconvoluted $[\mathrm{SDGRG}+\mathrm{H}]^{+}$(green) and $[\mathrm{GRGDS}+\mathrm{H}]^{+}$(red) using IM wave height voltage, wave velocity, and post-IM/CID acceleration voltage of $7.6 \mathrm{~V}, 300 \mathrm{~m} / \mathrm{s}$, and 40 $\mathrm{V}$, respectively. AT errors are reported at the 95\% C.L. $\left(\mathrm{n}_{\text {pure }}=5\right.$ and $\left.\mathrm{n}_{\text {decon }}=4\right)$. 


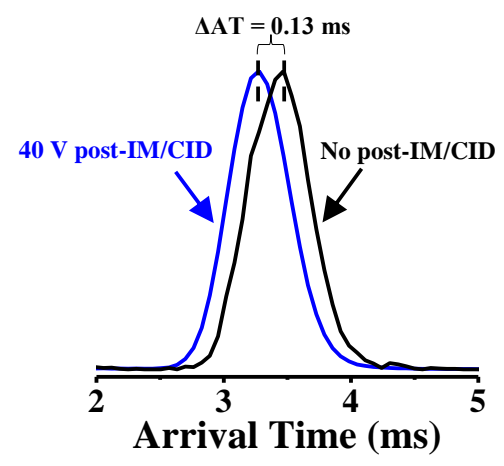

Figure 3. Unresolved IM profiles of an equimolar mixture of $[\mathrm{SDGRG}+\mathrm{H}]^{+}$and $[$GRGDS + $\mathrm{H}]^{+}(\mathrm{m} / z$ 491.22) using IM wave height and velocity of $7.6 \mathrm{~V}$ and $300 \mathrm{~m} / \mathrm{s}$, respectively, without post-IM/CID (black IM profile) and with post-IM/CID at $40 \mathrm{~V}$ acceleration voltage (blue IM profile). The arrival time shifts $(\Delta \mathrm{AT}=0.13 \mathrm{~ms})$ to a shorter value, due to post-IM/CID acceleration voltage.

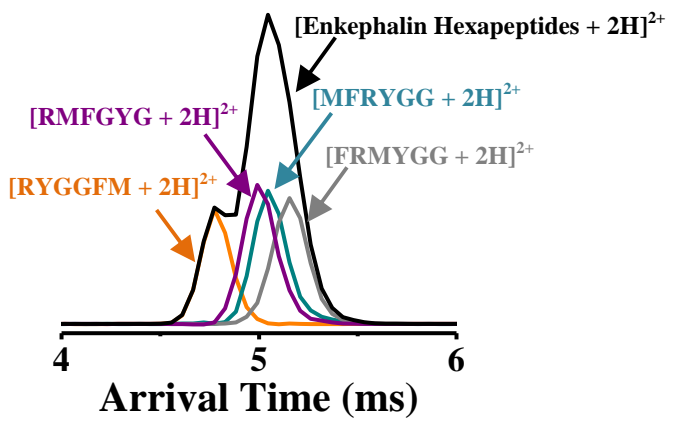

Figure 4. IM profiles of $[\mathrm{RYGGFM}+2 \mathrm{H}]^{2+}$ (orange), $[\mathrm{RMFGYG}+2 \mathrm{H}]^{2+}$ (purple), [MFRYGG $+2 \mathrm{H}]^{2+}\left(\right.$ cyan), and $[\text { FRMYGG }+2 \mathrm{H}]^{2+}$ (gray) before (black IM profile) and after (colored IM profiles) IM deconvolution at IM wave height voltage, wave velocity, and post-IM/CID acceleration voltage of $40 \mathrm{~V}, 1300 \mathrm{~m} / \mathrm{s}$, and $22 \mathrm{~V}$, respectively. 


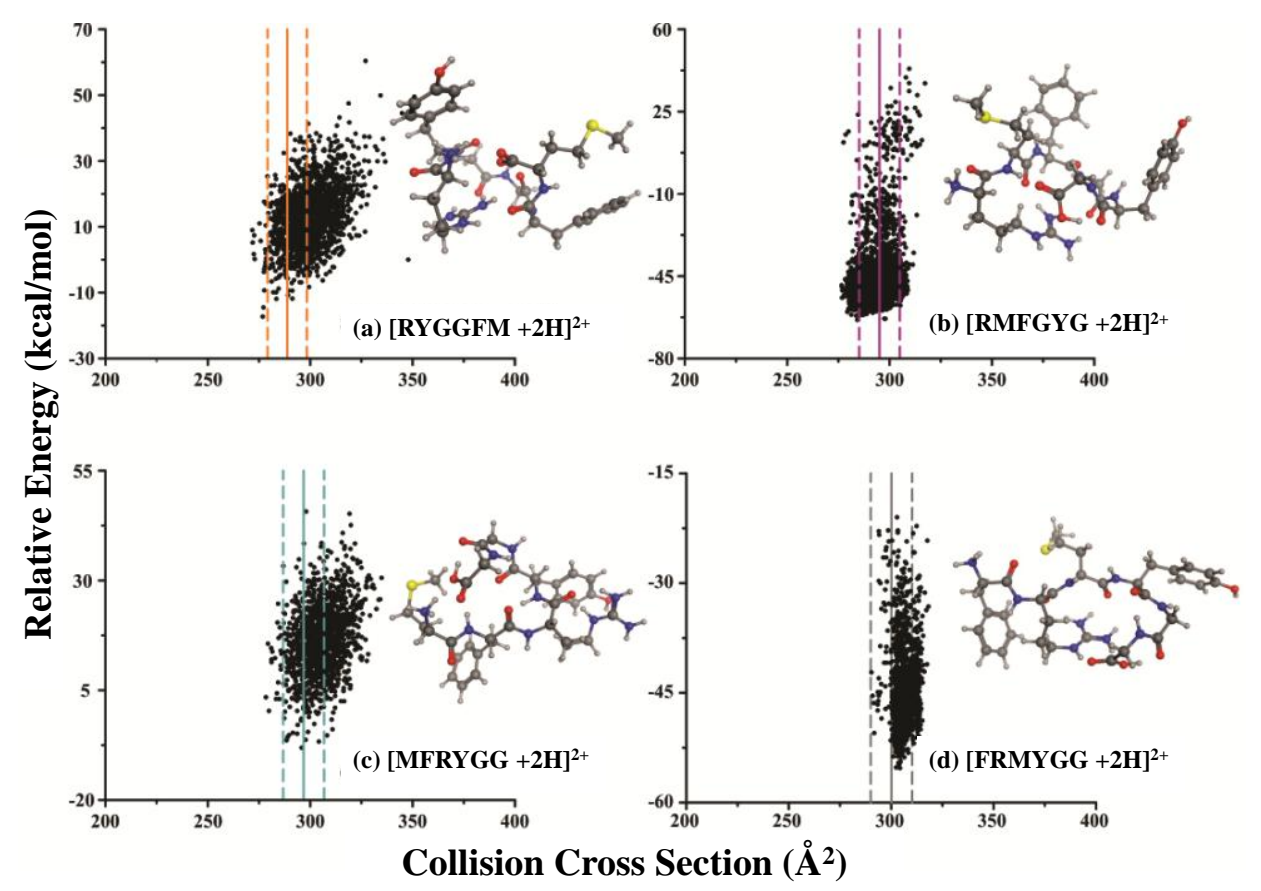

Figure 5. Computational results for calculating theoretical CCS values of the four enkephalin hexapeptides including (a) $[\mathrm{RYGGFM}+2 \mathrm{H}]^{2+}$, (b) $[\mathrm{RMFGYG}+2 \mathrm{H}]^{2+}$, (c) $[\mathrm{MFRYGG}+2 \mathrm{H}]^{2+}$, and (d) $[\text { FRMYGG }+2 \mathrm{H}]^{2+}$. Solid vertical lines on each plot represent the AT-corrected deconvoluted TWIM-MS CCS values and the associated 95\% C.L. are indicated with dashed lines; line colors match the corresponding deconvoluted IM profiles for hexapeptides in Figure 4. Representative conformations from RMSD clustering for each isomer are also included. Yellow, red, blue, gray, and white spheres represent sulfur, oxygen, nitrogen, carbon, and hydrogen atoms, respectively. 


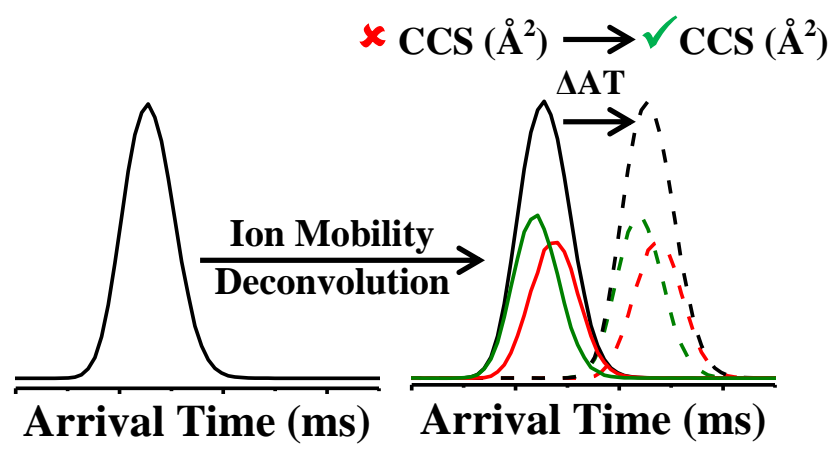

